\title{
Effects of Six-Month Aerobic Exercise Intervention on Sleep in Healthy Older Adults in the Brain in Motion Study: A Pilot Study
}

\author{
Veronica Guadagni ${ }^{\mathrm{a}, \mathrm{b}, \mathrm{e}, \mathrm{g}}$, Cameron M. Clark ${ }^{\mathrm{a}, \mathrm{b}, \mathrm{e}}$, Amanda Tyndall ${ }^{\mathrm{a}, \mathrm{b}, \mathrm{e}}$, Jill K. Raneri ${ }^{\mathrm{c}, \mathrm{d}}$, \\ Jillian S. Parboosingh ${ }^{\mathrm{i}, \mathrm{j}}$, David B. Hogan ${ }^{\mathrm{b}, \mathrm{c}, \mathrm{e}, \mathrm{g}}$, Patrick J. Hanly ${ }^{\mathrm{b}, \mathrm{c}, \mathrm{d}}$ and Marc J. Poulin ${ }^{\mathrm{a}, \mathrm{b}, \mathrm{e}, \mathrm{f}, \mathrm{g}, \mathrm{h}, *}$ \\ ${ }^{a}$ Department of Physiology and Pharmacology, Cumming School of Medicine, University of Calgary, \\ Calgary, Alberta, Canada \\ ${ }^{\mathrm{b}}$ Hotchkiss Brain Institute, Cumming School of Medicine, University of Calgary, Calgary, AB, Canada \\ ${ }^{\mathrm{c}}$ Department of Medicine, Cumming School of Medicine, University of Calgary, Calgary, AB, Canada \\ ${ }^{\mathrm{d}}$ Sleep Centre, Foothills Medical Centre, Calgary, AB, Canada \\ ${ }^{\mathrm{e}}$ Department of Clinical Neurosciences, Cumming School of Medicine, University of Calgary, \\ Calgary, AB, Canada \\ ${ }^{\mathrm{f}}$ Libin Cardiovascular Institute of Alberta, Cumming School of Medicine, University of Calgary, \\ Calgary, AB, Canada \\ $\mathrm{g}^{\mathrm{O}}$ 'Brien Institute for Public Health, Cumming School of Medicine, University of Calgary, \\ Calgary, AB, Canada \\ ${ }^{\mathrm{h}}$ Faculty of Kinesiology, University of Calgary, Calgary, AB, Canada \\ ${ }^{\mathrm{i}}$ Department of Medical Genetics, University of Calgary, Calgary, AB, Canada \\ ${ }^{\mathrm{j}}$ Alberta Children's Hospital Research Institute for Child and Maternal Health, Calgary, AB, Canada
}

Accepted 12 November 2018

\begin{abstract}
.
Background: Sleep disturbances have been shown to be associated with the presence of the apolipoprotein $(A P O E) \varepsilon 4$ allele, the well-known genetic risk factor for late-onset sporadic Alzheimer's disease (AD).

Objective: This study quantifies the effects of a six-month aerobic exercise intervention on objective and subjective sleep quality in middle-aged to older individuals including those at increased genetic risk for late-onset sporadic Alzheimer's disease (AD), who carry the apolipoprotein (APOE) $\varepsilon 4$ risk allele.

Methods: 199 sedentary men and women without significant cognitive impairments were enrolled in the Brain in Motion study, a quasi-experimental single group pre-test/post-test study with no control group. Participants completed a six-month aerobic exercise intervention and consented to genetic testing. Genotyping of $A P O E$ confirmed that 54 individuals were carriers of the $\varepsilon 4$ allele. Participants' subjective quality of sleep was assessed with the Pittsburgh Sleep Quality Index (PSQI) pre- and post-intervention. A convenience sample of participants $(n=29, A P O E \varepsilon 4+=7)$ consented to undergo two nights of in-home polysomnography (PSG) pre- and post intervention. Sleep architecture and respiratory variables were assessed.
\end{abstract}

\footnotetext{
*Correspondence to: Marc J. Poulin, PhD, DPhil, Laboratory of Human Cerebrovascular Physiology, Hotchkiss Brain Institute, Cumming School of Medicine, University of Calgary, Room 210-Heritage Medical Research Building, 3310 Hospital Drive
}

NW, Calgary, AB, T2N 4N1, Canada. Tel.: +1 403220 8372; E-mail: poulin@ucalgary.ca. 
Results: The six-month aerobic exercise intervention significantly improved participants' total PSQI score, sleep efficiency, and sleep latency in the full sample $(n=199)$. PSG results showed that total sleep time and sleep onset latency significantly improved over the course of the exercise intervention only in individuals who carried the APOE $\varepsilon 4$ allele. These results are, however, exploratory and need to be carefully interpreted due to the rather small number of APOE $\varepsilon 4+$ in the PSG subgroup. Conclusions: The six-month aerobic exercise intervention significantly improved participants' sleep quality with beneficial effects on PSG shown in individuals at increased genetic risk for late-onset sporadic AD.

Keywords: Alzheimer's disease, APOE $\varepsilon 4$, dementia, polysomnography

\section{INTRODUCTION}

The number of Canadians living with dementia is projected to double by 2031 [1]. There are currently no disease-modifying pharmacological therapies for Alzheimer's disease (AD), the most common cause of dementia among older individuals. The available pharmacological agents provide symptomatic benefit and have not been shown to address underlying disease processes [2-4]. Despite a large investment, drug development for $\mathrm{AD}$ has been marked by repeated failures [2-4]. This has led to a shift in prevention trials from targeting individuals already showing early cognitive symptoms, to interventions trials on individuals not yet showing symptom but who are at increased risk of $\mathrm{AD}$ [5].

Recent research has examined a possible link between changes in sleep quality and the development of AD pathology, particularly amyloid- $\beta$ deposition. One potential mechanism that could result in increased amyloid- $\beta$ deposition is the reduced activity of the glymphatic system when sleep is fragmented [6-8]. This system is involved in the clearance of metabolic waste products including amyloid- $\beta$ from the brain. This clearance takes place at a faster rate during sleep [8]. Reduced slow wave sleep (SWS), which typically occurs in older age has been considered as another potential mechanism through which sleep disruption influences the development of the pathological features of AD [9]. Improving sleep quality may be a strategy to delay the onset of $\mathrm{AD}$ and slow its progression [10].

The presence of the apolipoprotein $(A P O E) \varepsilon 4$ allele is a well-known genetic risk factor for lateonset sporadic AD [11], although the underlying mechanisms for this association are unsettled in the research literature $[12,13]$. The presence of this allele in older adults has also been associated with reduced sleep quality $[14,15]$. In a previous study [14], we examined the relationship between the quality of sleep and the presence of the APOE $\varepsilon 4$ allele.
Compared to non-carriers, individuals with the $\varepsilon 4$ allele had lower sleep efficiency (expressed as the ratio of total sleep time to the total time in bed), reduced total sleep time, and increased wakefulness after sleep onset (WASO) on polysomnography (PSG) but did not score differently on subjective (i.e., self-reported) measures of sleep. These results are consistent with previous literature showing the effects of the $\varepsilon 4$ allele on sleep architecture [16-20].

Exercise has been associated with improvements in general well-being, body weight and metabolism, cerebrovascular reserve, cognitive performance, and brain health (hippocampal volume and functional connectivity) [21-27]. Further, physical activity has been associated with improved sleep quality, particularly sleep efficiency, sleep latency and depth [28-30], possibly due to stimulation of the serotoninergic pathway [31]. Wilckens and colleagues [32] examined the role of sleep efficiency in mediating the effects of physical activity on cognitive functioning. They reported that improved sleep efficiency modulated the relationship between physical activity and improved cognitive performance. In another study [33], independent relationships between both physical activity (PA) and sleep efficiency and improvements in cognitive performance were found. Altogether, this evidence supports the hypothesis that aerobic exercise, partly by improving sleep quality, promotes better cognitive function and reduces the risk of developing sporadic late-onset AD.

In the current study, we examined the effects of a six-month aerobic exercise intervention on sleep quality in healthy middle-aged and older participants as an ancillary study of the larger Brain in Motion study [34], a quasi-experimental single group pretest/post-test study with no control group on the effects of aerobic exercise on cerebral blood flow and cognition. Our primary objective was to determine whether six-months of aerobic exercise improved overall sleep quality and sleep efficiency as determined by both subjective (self-reports) and objective 
(PSG) measures of sleep. As a secondary objective, we assessed if individuals who carried the risk allele $A P O E \varepsilon 4$ showed differential effects with exercise on sleep quality compared to non-carriers. Specifically, we hypothesize that individuals carrying at least one $\varepsilon 4$ allele $(A P O E \varepsilon 4+)$ would show greater improvements in sleep quality as compared to non-carriers.

\section{METHODS}

\section{Participants}

Two hundred and sixty-five sedentary middleaged to older participants of either sex (mean age (SD) 65.2 (6.4) years; 141 (53.2\%) females) without significant cognitive impairment (mean Montreal Cognitive Assessment (MoCA) [35] score (SD) 27.2 (1.6)) were enrolled in the Brain in Motion study, a quasi-experimental single group pre-test/post-test study with no control group [34]. A total of 203 individuals completed the exercise intervention with 62 dropouts (mainly due to changes in their medical conditions). Of the 203 who completed the intervention, 199 consented to genetic testing and their data are reported in this manuscript.

Participants were volunteers recruited through word-of-mouth, social media, posters and newspaper advertisements. Ethics approval was obtained by the University of Calgary Conjoint Health Research Ethics Board (CHREB: REB 14-2284) and the study was done in agreement with the Helsinki Declaration of 1975. Participants provided informed written consent prior to enrollment and a separate written consent for genetic testing. For further details, please see Tyndall et al. [34].

\section{APOE genotyping}

Genomic DNA was extracted from buffy coats obtained from whole blood samples (Gentra Puregene Blood Kit; Qiagen, Venlo, Netherlands). Samples underwent PCR amplification followed by Sanger sequencing (Big Dye v1.1 Cycle Sequencing Kit; Applied Biosystems, Foster City, CA) on ABI 3130XL Genetic Analyzer (Applied Biosystems). Mutation Surveyor DNA Variant Analysis software (Soft Genetics, LLC; State College, PA) was identify $A P O E \varepsilon 2, \varepsilon 3$, and $\varepsilon 4$ alleles by manually combining the alleles from the single nucleotide polymorphisms NM_000041.2:c.388T>C (p.(Cys130Arg); rs429358) and c.526C $>$ T (p.(Arg176Cys); rs7414). Individuals who carried the $\varepsilon 3 / \varepsilon 4, \varepsilon 2 / \varepsilon 4$, and $\varepsilon 4 / \varepsilon 4$
Table 1

Detailed breakdown of the $A P O E$ alleles in $\mathrm{N}=199$ individuals

\begin{tabular}{lc}
\hline APOE alleles & $n$ \\
\hline$\varepsilon 3 / \varepsilon 3$ & 123 \\
$\varepsilon 4 / \varepsilon 4$ & 5 \\
$\varepsilon 2 / \varepsilon 2$ & 1 \\
$\varepsilon 2 / \varepsilon 4$ & 4 \\
$\varepsilon 2 / \varepsilon 3$ & 21 \\
$\varepsilon 3 / \varepsilon 4$ & 45 \\
TOT & 199 \\
\hline
\end{tabular}

allele combinations were classified as APOE \&4+, while individuals who carried the other allele combinations $(\varepsilon 2 / \varepsilon 2, \varepsilon 2 / \varepsilon 3, \varepsilon 3 / \varepsilon 3)$ were classified as $A P O E$ \&4- (detailed breakdown of the APOE alleles is reported in Table 1$)$. The $\varepsilon 2 / \varepsilon 4$ allele combination has been shown to have protective effects [36] however we included the $\varepsilon 2 / \varepsilon 4$ carriers in the $A P O E$ $\varepsilon 4+$ group because of a recent meta-analysis of clinical and autopsy-based studies on sporadic late onset $\mathrm{AD}$ reporting higher risk of $\mathrm{AD}$ in individuals with one copy of the $\varepsilon 4$ allele $(\varepsilon 2 / \varepsilon 4$, OR $2.6 ; \varepsilon 3 / \varepsilon 4$, OR $3.2)$ or two copies ( $\varepsilon 4 / \varepsilon 4$, OR 14.9$)$ among Caucasian subjects [37].

One hundred and ninety-nine participants (mean age $(\mathrm{SD})=65.00(6.00)$ years; mean years of formal education $(\mathrm{SD})=15.8(2.5)$ years; mean MoCA $(\mathrm{SD})=27.5(1.4), \mathrm{BMI}(\mathrm{SD})=27$ (4) $\mathrm{kg} \cdot \mathrm{m}^{-2} ; 103$ $(51.8 \%)$ females) completed the exercise intervention, had complete PSQI data, and underwent genetic testing. Fifty-four individuals were APOE $\varepsilon 4+$ (mean age $(\mathrm{SD})=64.5$ (5.9) years; mean years of formal education $(\mathrm{SD})=16.0$ (2.3) years; mean MoCA $(\mathrm{SD})=27.5$ (1.4); 24 (44.4\%) females).

\section{Experimental procedures}

Participants completed four testing sessions including one just before the start of the six-month exercise intervention (taken as the baseline assessment for this report) and one at the end. At each session, participants were asked to complete the Pittsburgh Sleep Quality Index (PSQI) [38], a retrospective self-report measure assessing their quality of sleep during the month preceding the study. Scores for the seven components of the PSQI were computed and represented 1) subjective sleep quality; 2) sleep latency; 3) sleep duration; 4) sleep efficiency; 5) sleep disturbance; 6) sleep medications; and, 7) daytime dysfunction. The component scores were then summed to produce a global score (range 0 to 21). Lower scores indicate better sleep with global scores 
$\geq 5$ indicating poor sleep quality. Participants also completed the Epworth Sleepiness Scale (ESS) [39] at each session, which measures individuals' general level of daytime sleepiness. In this study, we only report pre- and post- intervention data for participants that 1) completed the exercise intervention, 2) provided pre/post PSQI data, and 3) consented to genetic testing.

\section{In-home polysomnography (PSG)}

A convenience sample of participants $(n=29$, APOE $\varepsilon 4+=7$ ) consented to undergo two nights of unattended PSG recordings in their home, which were done just prior to six-month exercise intervention and immediately after its conclusion. This was a convenience sample and post-hoc evaluations revealed matching pre-intervention characteristics to the full sample (i.e., mean age $(\mathrm{SD})=65.00$ (5.00) years; mean years of formal education $(\mathrm{SD})=16.7$ (3.09) years; mean $\mathrm{MoCA}=27.5(1.4)$; $\mathrm{BMI}(\mathrm{SD})=26.4$ (3.8) $\mathrm{kg} \cdot \mathrm{m}^{-2} ; 17$ (58.6\%) females). We used the Embletta MPR PG (Natus Medical Inc., Pleasanton, CA) and an ST1 proxy unit. Each participant was instrumented by a trained technician in his/her home. Respiratory data were obtained through finger pulse oximetry and respiratory effort through inductive plethysmography. Airflow was measured by using nasal pressure and an oro-nasal thermistor. Participants were also instrumented with standard one-lead ECG. Body position was derived using the gravity sensors in the Embletta unit. Electrode placement for the EEG followed the standard 10-20 system. Data were scored by an experienced registered polysomnographic technologist (J.K.R.). The following variables were extracted: 1) total sleep time; 2) total recording time; 3) sleep efficiency; 4) sleep onset latency; 5) REM onset latency; and, 6) number of awakenings. The number of minutes spent in each sleep stage (Awake, N1, N2, N3, REM) was also computed together with respiratory variables (including oximetry) that were then used to compute the apnea/hypopnea index (AHI). Apneas and hypopneas were defined according to the revised American Academy of Sleep Medicine guidelines [40].

\section{Six-month aerobic exercise intervention}

Participants were enrolled in a supervised sixmonth aerobic exercise training program held three days per week at the Fitness Centre in the Faculty of Kinesiology at the University of Calgary.
Each session included a five-minute warm up, aerobic exercise and a five-minute cool down with stretching. Exercise prescriptions followed the American College of Sports Medicine guidelines [41]. As participants progressed through the program, the duration of exercise between warm up and cool down increased from 20 to 40 minutes. In the case of aerobic exercise, the intensity was determined based on individual $\mathrm{VO}_{2}$ max. The intensity increased from 30-45\% maximum heart rate reserve (HRR) to 60-70\%. For further details, see Tyndall et al. [34].

\section{Statistical analysis}

Data were analyzed using IBM SPSS Statistics for Windows, version 24.0 (IBM, Armonk, NY, USA). Pre- intervention descriptive statistics are reported in Tables 2 and 3; Paired samples $t$-tests were used to compare pre- and post- measurements for continuous variables and descriptive statistics are reported in Tables 4 and 5. Categorical variables were examined with the McNemar Chi-squared for repeated measures. Further, stratified analyses for genetic status were conducted. All analyses were two-tailed and

Table 2

Pre- intervention subjective measures of sleep (PSQI) in individuals with APOE $\varepsilon 4 \pm$ alleles (Means and SD)

\begin{tabular}{lccc}
\hline PSQI & $\begin{array}{c}\text { APOE } \varepsilon 4+ \\
(n=54) \\
\text { PRE }\end{array}$ & $\begin{array}{c}\text { APOE } \varepsilon 4- \\
(n=145)\end{array}$ & \\
& PRE & $p$ \\
\hline Global score & $5.8(3.2)$ & $5.8(3.1)$ & 0.958 \\
Sleep efficiency (\%) & $84.3(10.7)$ & $85.3(10.1)$ & 0.554 \\
Sleep latency (min) & $17.5(15.5)$ & $18.5(17.2)$ & 0.715 \\
Sleep duration (min) & $411.5(61.3)$ & $420.0(56.3)$ & 0.262 \\
\hline
\end{tabular}

PSQI, Pittsburgh Sleep Quality Index; SD, standard deviation.

Table 3

Pre- intervention objective measures of sleep (PSG) in individuals with APOE $\varepsilon 4 \pm$ alleles (Means and SD)

\begin{tabular}{lccc}
\hline PSG & $\begin{array}{c}A P O E \varepsilon 4+ \\
(n=7)\end{array}$ & $\begin{array}{c}\text { APOE } \varepsilon 4- \\
(n=22)\end{array}$ & \\
& PRE & PRE & $p$ \\
\hline Total sleep time (min) & $352(67)$ & $441(50)$ & 0.001 \\
Total recording time (min) & $481.1(25.6)$ & $502.6(61.1)$ & 0.378 \\
Sleep onset latency (min) & $20.8(25.6)$ & $14.7(13.4)$ & 0.414 \\
Sleep efficiency (\%) & $74(17)$ & $88(3)$ & 0.001 \\
Stage N1(min) & $52(23)$ & $68(30)$ & 0.219 \\
Stage N2 (min) & $207(48)$ & $281(30)$ & $<0.001$ \\
Stage N3 (min) & $15(16)$ & $13(16)$ & 0.783 \\
REM (min) & $78(17)$ & $79(18)$ & 0.863 \\
REM onset latency (min) & $86(23)$ & $114(65)$ & 0.270 \\
Number of awakenings & $28(11)$ & $28(6)$ & 0.824 \\
\hline
\end{tabular}

PSG, polysomnography; REM, rapid eye movements; SD, standard deviation. 
Table 4

Subjective measures of sleep (PSQI) and BMI in individuals with APOE $\varepsilon 4 \pm$ alleles (Means and SD)

\begin{tabular}{|c|c|c|c|c|c|c|}
\hline & \multicolumn{3}{|c|}{$A P O E \& 4+(n=54)$} & \multicolumn{3}{|c|}{$A P O E \varepsilon 4-(n=145)$} \\
\hline & PRE & POST & $p$ & PRE & POST & $p$ \\
\hline PSQI Global score & $5.8(3.2)$ & $5.5(3.2)$ & 0.181 & $5.8(3.1)$ & $5.4(3.0)$ & 0.004 \\
\hline Sleep efficiency $(\%)$ & $84.3(10.7)$ & $88.1(14.3)$ & 0.021 & $85.4(10.0)$ & $87.6(11.1)$ & 0.003 \\
\hline Sleep latency ( $\mathrm{min})$ & $17.9(15.7)$ & $15.4(11.8)$ & 0.095 & $18.7(17.5)$ & $16.4(16.2)$ & 0.049 \\
\hline Sleep duration (min) & $411.3(60.5)$ & $401.8(63.1)$ & 0.062 & $421.6(55.2)$ & $419.5(59.8)$ & 0.531 \\
\hline $\operatorname{BMI}\left(\mathrm{kg} \cdot \mathrm{m}^{-2}\right)$ & $26.5(3.4)$ & $26.1(3.4)$ & $<0.001$ & $27.1(3.8)$ & $26.8(3.7)$ & $<0.001$ \\
\hline
\end{tabular}

PSQI, Pittsburgh Sleep Quality Index; SD, standard deviation; BMI, body mass index.

Table 5

Objective measures of sleep (PSG) in individuals with APOE $\varepsilon 4 \pm$ alleles (Means and SD)

\begin{tabular}{|c|c|c|c|c|c|c|}
\hline \multirow[b]{2}{*}{ PSG } & \multicolumn{3}{|c|}{$A P O E \varepsilon 4+(n=7)$} & \multicolumn{3}{|c|}{$A P O E \varepsilon 4-(n=22)$} \\
\hline & PRE & POST & $p$ & PRE & POST & $p$ \\
\hline Total sleep time (min) & $352(67)$ & $406(44)$ & 0.008 & $441(50)$ & $423(63)$ & 0.187 \\
\hline Sleep onset latency ( $\mathrm{min})$ & $20.8(25.6)$ & $15.3(23.7)$ & 0.010 & $14.7(13.4)$ & $10.5(6.5)$ & 0.169 \\
\hline Sleep efficiency $(\%)$ & $74(17)$ & $83(11)$ & 0.040 & $88(3)$ & $88(8)$ & 0.872 \\
\hline Stage N1(min) & $52(23)$ & $55(32.8)$ & 0.693 & $68(30)$ & $57(23)$ & 0.053 \\
\hline Stage N2 (min) & $207(48)$ & $240(42)$ & 0.088 & $281(30)$ & $280(37)$ & 0.937 \\
\hline Stage N3 (min) & $15(16)$ & $14(14)$ & 0.808 & $13(16)$ & $11(13)$ & 0.443 \\
\hline REM (min) & $78(17)$ & $97(37)$ & 0.095 & $79(18)$ & $75(29)$ & 0.552 \\
\hline REM onset latency (min) & $86(23)$ & $78(30)$ & 0.603 & $114(65)$ & $115(58)$ & 0.928 \\
\hline Number of awakenings & $28(11)$ & $26(9)$ & 0.549 & $28(6)$ & $28(10)$ & 0.919 \\
\hline
\end{tabular}

PSG, polysomnography; REM, rapid eye movements; SD, standard deviation.

statistical significance was set at $p<0.05$, BonferroniHolm correction for multiple comparisons was used [42]. We performed four tests for the PSQI in the full sample $(n=199)$ looking at PSQI total score, sleep efficiency, duration, and sleep latency. According to the Bonferroni-Holm method [42] $\alpha=0.05 /$ $(4-1+1)=0.0125$ for the smallest $p$ value, $\alpha=0.05 /(4-$ $2+1)=0.0167$ for the second smallest, $\alpha=0.05 /(4-3$ $+1)=0.025$ for the third and $\alpha=0.05 /(4-4+1)=0.05$ for the last. In the PSG, we examined similar characteristics of sleep quality and performed three tests looking at total sleep time, sleep efficiency, and sleep latency. Similarly, as per the Bonferroni-Holm method [42] $\alpha=0.05 /(3-1+1)=0.016$ for the smallest $p$ value, $\alpha=0.05 /(2-2+1)=0.025$ for the second smallest, $\alpha=0.05 /(3-3+1)=0.05$ for the last.

\section{RESULTS}

\section{Subjective sleep measures}

One-hundred and eleven participants (55.7\%) out of 199 reported global scores on the PSQI $\geq 5$ indicating poor sleep quality at pre-intervention. This percentage decreased to $46.7 \%$ (93/199, $9 \%$ decrease,) at post-intervention. Statistically significant but modest (6.9\%) improvements in global PSQI scores between pre- $($ mean $(\mathrm{SD})=5.8(3.1))$ and post-intervention $($ mean $(\mathrm{SD})=5.4(3)), t_{198}=3.166$, $p=0.002$, Cohen's $d_{z}=0.22$; see Fig. 1A) were found. Statistically significant but likewise modest (3.4\%) improvements were found in sleep efficiency (pre-intervention mean $(\mathrm{SD})=85.1(10.2)$ versus post-intervention mean $(\mathrm{SD})=88 \quad(12)$, $t_{195}=-3.845, p<0.001$, Cohen's $\mathrm{d}_{\mathrm{z}}=0.27$; see Fig. 1B). Sleep latency also showed a statistically significant decrease of $14.1 \%$ post-intervention (preintervention mean $(\mathrm{SD})=19$ (17) minutes versus post-intervention mean $(\mathrm{SD})=16.3(15.1)$ minutes), $t_{190}=2.596, p=0.010$, Cohen's $\mathrm{d}_{\mathrm{z}}=0.18$; see Fig. 1C). No statistically significant changes were found in sleep duration $(p=0.154)$. Among the four categorical components statistically significant differences $(p=0.012)$ were only found for the subjective sleep quality component. Sixty percent of participants reported very good sleep quality after the intervention compared to $51.3 \%$ before. Changes in sleep quality were not related to changes in body mass index. There were no statistically significant changes in daytime sleepiness as measured by the $\operatorname{ESS}(p=0.442)$.

The analysis of pre-intervention characteristics revealed no statistically significant differences in global PSQI score, sleep efficiency, sleep latency and sleep durations between APOE $\varepsilon 4$ carriers and non-carriers (see Table 2). Over the course of the exercise intervention, statistically significant differences in global PSQI scores were only 

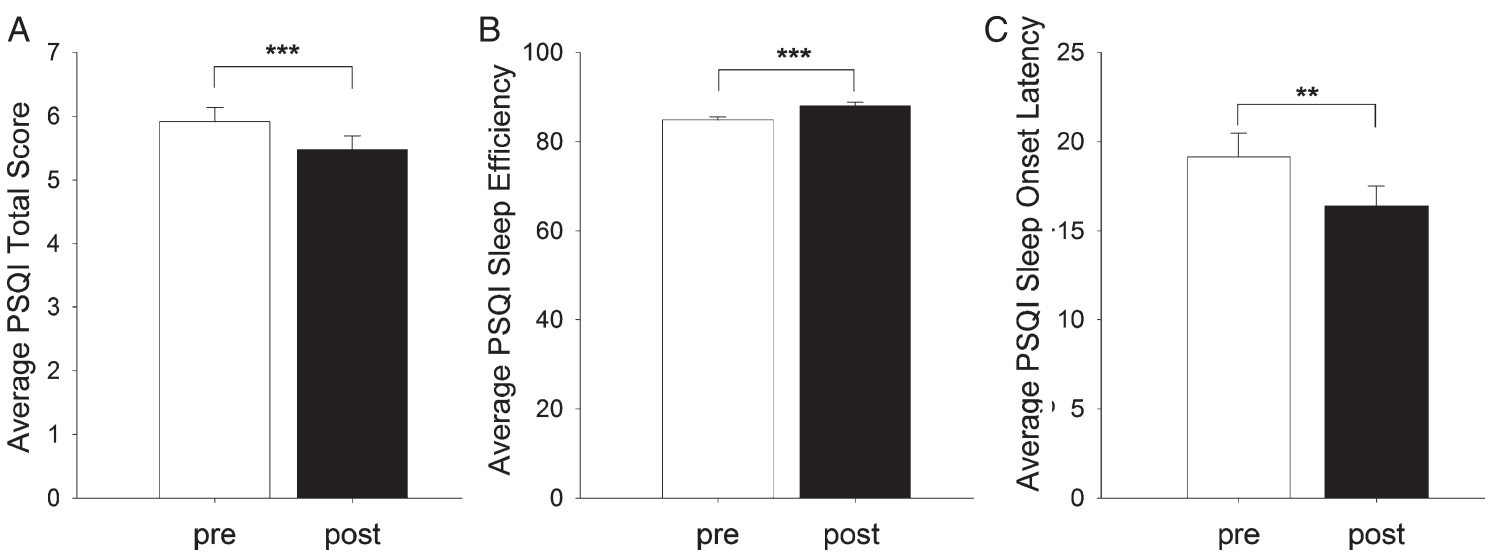

Fig. 1. Effects of six-months aerobic exercise on subjective sleep measures (PSQI). Panel A illustrates the total Pittsburgh Sleep Quality Index (PSQI) in the overall sample $(n=199)$ between pre-intervention scores [Mean $(\mathrm{SD})=5.8(3.1)$ ] and post-intervention scores $[\mathrm{Mean}$ $(\mathrm{SD})=5.4(3)]$. We note a significant decrease in the total PSQI score, reflecting an 6.9\% improvement in sleep quality. Panel B illustrates sleep efficiency in the overall sample $(n=199)$ between pre-intervention [Mean $(\mathrm{SD})=85.1(10.2)]$ and the end of the exercise intervention $[$ Mean $(\mathrm{SD})=88(12)]$. We note a significant $3.4 \%$ increase in sleep efficiency in the overall sample. Panel C illustrates a $14.1 \%$ decrease in sleep latency in the overall sample $(n=199)$ before (pre-intervention) [Mean (SD) $=19$ (17) minutes] and after (post-intervention) sixmonths of aerobic exercise intervention [Mean $(\mathrm{SD})=16.3$ (15.1) minutes]. Bars represent Means and Standard Error; ${ }^{*} p<0.5 ;{ }^{* *} p<0.01$; ${ }^{* * *} p<0.001$.

A

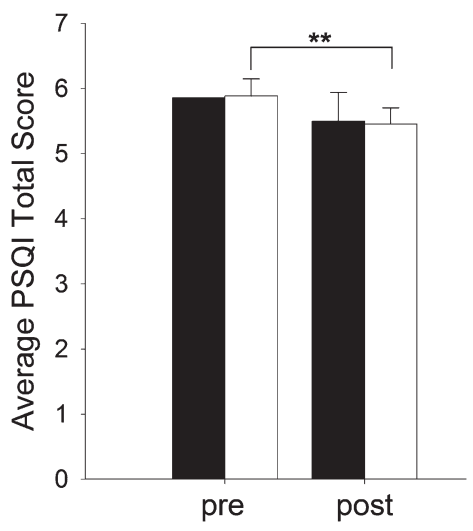

B

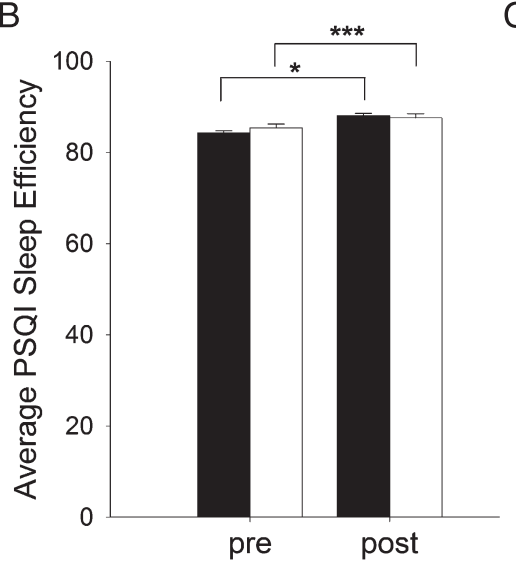



Fig. 2. Effects of a 6-month aerobic exercise on subjective sleep quality and sleep efficiency in individuals with $A P O E \varepsilon 4 \pm$ alleles. Panel A highlights changes before (pre-intervention) $[\mathrm{Mean}(\mathrm{SD})=5.8(3.1)]$ and after (post-intervention) six-months of aerobic exercise intervention [Mean (SD) = 5.4 (3.0)], in total Pittsburgh Sleep Quality Index (PSQI) in the APOE $\varepsilon 4$ - (light bars, $n=145$ ) group but not in the $A P O E \varepsilon 4+$ (dark bars, $n=54$ ) group. Panel B shows improvements in sleep efficiency in both APOE $\varepsilon 4+$ (dark bars, $n=54)$ and APOE $\varepsilon 4-$ (light bars, $n=145)$ groups, with the $A P O E \varepsilon 4+$ group having the greatest improvement $(4.5 \%)$ before (pre-intervention $[\mathrm{Mean}(\mathrm{SD})=84.3(10.7)]$ and after (post-intervention) six-months of aerobic exercise intervention Mean $(\mathrm{SD})=88.1$ (14.3). Panel C shows a decrease in sleep latency only in the APOE $\varepsilon 4$ - (light bars, $n=145$ ) group from pre-intervention [Mean $(\mathrm{SD})=18.7(17.5)$ ] to post-intervention $[\mathrm{Mean}(\mathrm{SD})=16.6$ (16.2)]. Bars represent Means and Standard Error; ${ }^{*} p<0.5$; $^{* *} p<0.01 ;{ }^{* * *} p<0.001$.

found in $A P O E$ \&4- individuals (pre-intervention mean $(\mathrm{SD})=5.8(3.1)$ versus post-intervention mean $(\mathrm{SD})=5.4$ (3.0) $, t_{144}=2.889, p=0.004$, Cohen's $\mathrm{d}_{\mathrm{z}}=0.23$; see Fig. $2 \mathrm{~A}$ ) with no significant differences found in the APOE $\varepsilon 4+$ group $(p=0.181)$. Statistically significant differences were found in sleep efficiency for both $A P O E \varepsilon 4+$ and $A P O E \varepsilon 4-$ participants (pre-intervention $A P O E \varepsilon 4+$ mean $(\mathrm{SD})=84.3$
$(10.7) / A P O E \quad \varepsilon 4-$ mean $(\mathrm{SD})=85.4(10.0)$ versus post-intervention $A P O E \quad \varepsilon 4+$ mean $(\mathrm{SD})=88.1$ (14.3)/APOE $\varepsilon 4-$ mean (SD) = 87.6 (11.1); (APOE $\varepsilon 4+: t_{53}=-2.390, p=0.021,4.5 \%$ change, Cohen's $\mathrm{d}_{\mathrm{z}}=0.32$ APOE $\varepsilon 4-: t_{142}=-3.011, p=0.003,2.5$ $\%$ change, Cohen's $d_{z}=0.25$; see Fig. $2 B$ ). Sleep latency decreased only in $A P O E$ \&4- participants (pre-intervention mean $(\mathrm{SD})=18.7$ (17.5) minutes 

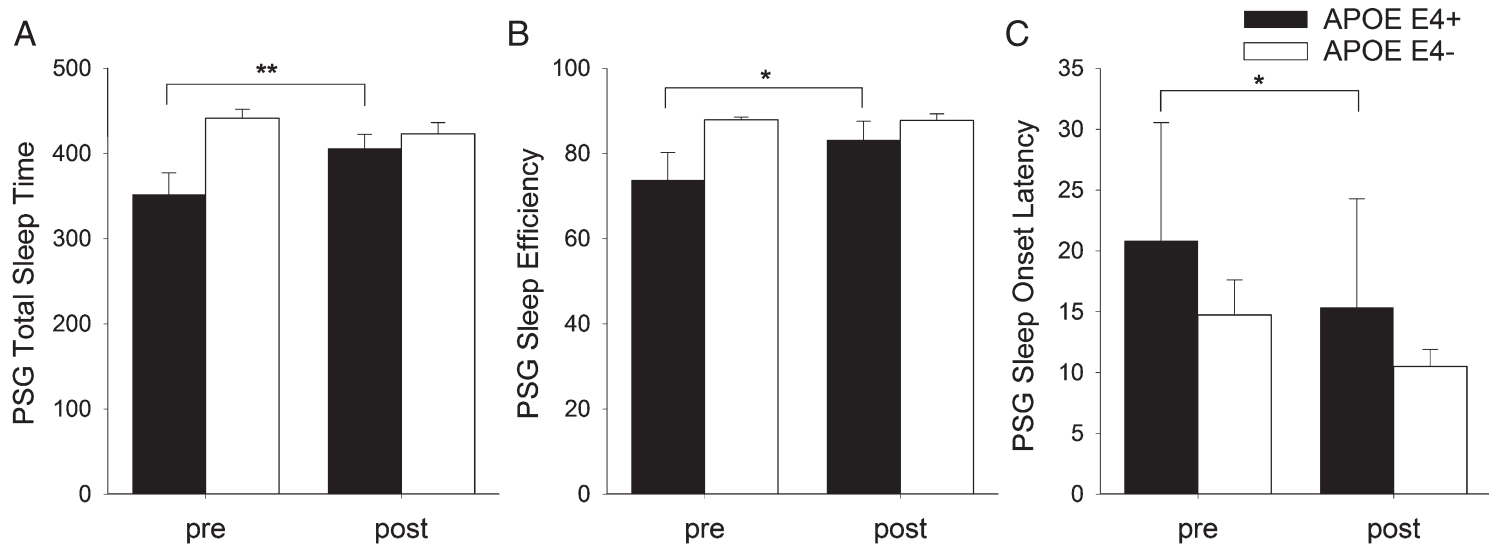

Fig. 3. Effects of a 6-month aerobic exercise on objective sleep measures (PSG) in individuals with APOE $\varepsilon 4 \pm$ alleles. Panel A highlights changes in total sleep time before (pre-intervention) [Mean $(\mathrm{SD})=352$ (66.7) minutes] and after (post-intervention) six-months of aerobic exercise intervention [Mean (SD) $=406$ (44.2) minutes] in the APOE $\varepsilon 4+($ dark bars, $n=7)$ group but not in the APOE $\varepsilon 4-($ light bars, $n=22)$ group. Panel B shows changes in sleep efficiency before (pre-intervention) [Mean $(\mathrm{SD})=73.8(16.9)]$ and after (post-intervention) [Mean $(\mathrm{SD})=83.2$ (11.4)] six-months of aerobic exercise intervention in the APOE $\varepsilon 4+$ (dark bars, $n=7$ ) group but not in the APOE $\varepsilon 4-$ (light bars, $n=22$ ) group. Panel $\mathrm{C}$ shows changes in sleep onset latency before (pre-intervention) [Mean $(\mathrm{SD})=20.8(25.6)]$ ] and after (post-intervention) [Mean $(\mathrm{SD})=15.3(23.7)$ ] six-months of aerobic exercise intervention in the APOE $\varepsilon 4+$ (dark bars, $n=7)$ group but not in the APOE $\varepsilon 4-$ (light bars, $n=22$ ). Bars represent Means and Standard Error; ${ }^{*} p<0.5 ;{ }^{* *} p<0.01 ;{ }^{* * *} p<0.001$.

versus post-intervention mean $(\mathrm{SD})=16.6(16.2)$ minutes, $t_{138}=1.982, p=0.049$, Cohen's $\mathrm{d}_{\mathrm{z}}=0.16$ ) (see Fig. 2C). However, this comparison did not survive the Bonferroni-Holm correction for multiple comparisons $\alpha=0.05 /(4-3+1)=0.025)$. No significant changes were found in sleep latency for $A P O E$ $\varepsilon 4+$ or sleep duration for either group.

\section{Polysomnography}

There were no statistically significant differences in PSG variables over the course of the exercise intervention in the full sample. The analysis of pre- intervention characteristics revealed statistically significant differences at pre-intervention between $A P O E \& 4$ carriers and non-carriers in total sleep time ( $p=0.001)$, sleep efficiency $(p=0.001)$, and N2 sleep stage ( $p=<0.001$, see Table 3$)$ with APOE $\varepsilon 4$ carriers reporting less total sleep time, worse sleep efficiency and less minutes spent in N2 stage of sleep. Over the course of the exercise intervention, stratified analyses revealed statistically significant improvements exclusively in participants who carried the $\varepsilon 4$ allele. There were statistically significant differences in total sleep time (pre-intervention APOE $\varepsilon 4+$ mean $(\mathrm{SD})=352$ (66.7) minutes versus post-intervention mean $(\mathrm{SD})=406(44.2)$ minutes, $t_{6}=-3.880 p=0.008$, Cohen's $d_{z}=1.47$; see Fig. $3 \mathrm{~A}$ ) and sleep efficiency (pre-intervention APOE $\varepsilon 4+$ mean $(\mathrm{SD})=73.8 \quad(16.9)$ versus post-intervention (mean $(\mathrm{SD})=83.2(11.4)], t_{6}=-2.615, p=0.040$, Cohen's $d_{z}=0.99$; see Fig. 3B), however, this comparison did not survive the Bonferroni-Holm correction for multiple comparisons $(\alpha=0.05 /(2$ $2+1)=0.025$ ). Sleep onset latency in $A P O E \varepsilon 4+$ significantly changed from pre- to post- intervention (pre-intervention APOE $\varepsilon 4+$ mean $(\mathrm{SD})=20.8(25.6)$ versus post-intervention mean $(\mathrm{SD})=15.3(23.7)$, $t_{6}=3.702, p=0.010$, Cohen's $d_{z}=1.38$; see Fig. 3 C). These changes were not related to changes in AHI ( $p=0.710, p=0.118, p=0.353$ for total sleep time, sleep efficiency and sleep onset latency, respectively).

\section{DISCUSSION}

We report on the effects of a six-month aerobic exercise intervention on both subjective (PSQI) and objective (PSG) measures of sleep quality in healthy middle-aged and older adults with and without the $A P O E \varepsilon 4$ risk allele. Major findings from the PSQI data for the entire study sample were an improvement in sleep quality reflected by a decrease in overall PSQI scores after the exercise intervention, reduced sleep latency, and increased sleep efficiency. The changes seen in sleep quality were modest in nature though statistically significant. No significant difference in terms of percent change was found between carriers and non-carriers, which is similar to other 
studies [43]. PSG data showed statistically significant improvements in total sleep time and sleep onset latency exclusively in the individuals with the $\varepsilon 4$ allele. The changes in sleep efficiency in APOE $\varepsilon 4+$ did not survive correction for multiple comparisons. These results are exploratory and need to be carefully interpreted due to the rather small number of participants with $A P O E \varepsilon 4+$ in the PSG subgroup.

Previous literature on sleep disturbances in individuals who carry the risk allele had shown worse sleep quality $[14,16]$. Yesavage and colleagues [16] conducted a longitudinal study on 40 communitydwelling older adults using actigraphy and found that $A P O E \varepsilon 4+$ carriers had worse sleep efficiency and greater WASO compared to non-carriers. A study from our laboratory [14] showed that carriers had shorter total sleep time, lower sleep efficiency, longer sleep onset latency, greater WASO and less time spent in Stage 2 sleep. To date, no study examined the effects of a lifestyle intervention such as aerobic exercise on the sleep quality of individuals at increased genetic risk of developing AD. Our study suggests that aerobic exercise might improve sleep in these individuals.

We collected subjective measures of sleep with the PSQI, and objective measures of sleep with the gold standard PSG. Our data show limited association between objective and subjective measures of sleep. Specifically, the only significant correlations were found between the change in sleep efficiency over the course of the exercise intervention from the PSG and global PSQI postintervention scores $(r=0.454, p=0.039)$ and sleep efficiency pre-intervention scores from the PSG and pre-intervention global PSQI scores $(r=0.426$, $p=0.030$ ). Further, we found differential effects of the exercise intervention between the PSQI and the PSG results. Overall these findings indicate a lack of association between subjective and objective measures of sleep as previously shown in other studies [44]. One possible explanation may be the differences in sample sizes and composition between individuals who completed the PSQI $(n=199)$ and individuals who underwent PSG recordings $(n=29)$. Another potential explanation is that the PSQI reflects the individual perception of his/her own sleep rather than an objective evaluation of the sleep [45]. This may be especially problematic in an older population who have grown accustomed to diminished sleep quality and perceive it as "normal" [46]. Further studies are necessary to replicate our findings in larger samples.
This study has several limitations. Neither participants nor investigators were blinded to the intervention. The small sample size, especially for PSG with only seven individuals carrying the risk allele limits the robustness and generalizability of our results. The sample size also limited the choice and power of the statistical analyses. Furthermore, our sample was healthy with an overall good quality of sleep as measured by the PSQI. Although we found a statistically significant positive change in global PSQI scores with the exercise intervention, this change cannot be defined as a clinically meaningful change according to the method discussed by Surman et al. [47]. Moreover, the lack of a control group in the study limits our ability to make firm conclusions about the effect of the exercise intervention. Other factors such as increased socialization may have led to the improvements in general wellbeing, mood, and sleep. Our results are preliminary and should be interpreted with caution. Future studies are needed to confirm and expand on our findings. We did not collect a familiarization night for PSG and therefore participants scores may reflect the discomfort of sleeping with unfamiliar equipment. Finally, the presence of sleep complaints was not an inclusion criterion. We cannot comment on the potential impact of the exercise intervention on sleep in a population with greater sleep disturbances at baseline.

In our study, aerobic exercise failed to show a clinically meaningful change in the quality of sleep of generally healthy older adults with no sleep complaints at baseline. However, our results could inform the design of future studies targeting older individuals with sleep complaints, a greater degree of comorbidities and/or at higher risk for AD that could shed light on the potential mediating role of improved sleep in understanding the relationship between exercise and cognitive status.

\section{ACKNOWLEDGMENTS}

This is an ancillary study of the Brain in Motion Study, funded by Canadian Institutes of Health Research (CIHR) and The Brenda Strafford Foundation Chair in Alzheimer Research (BSFCAR). V.G. was supported by The Brenda Strafford Centre on Aging, within the O'Brien Institute for Public Health, the BSFCAR and the Alzheimer's Society of Canada Postdoctoral Fellowship. C.M.C. was supported by the T. Chen Fong Post-Doctoral Fellowship in Medical Imaging Science, A.V.T. was supported by an 
Alzheimer Society of Canada Doctoral Award and the BSFCAR. J.K.R. was supported by funds from a Heart and Stroke Foundation of Canada Grant-inAid (PI M.J.P., co-applicant P.J.H.). M.J.P. holds the BSFCAR. Funding for the study and all biochemical analyses were provided by CIHR (PI M.J.P.) and the BSFCAR. The funders played no role in the concept and design of this study, analysis or interpretation of the data, or drafting and critical revision of the manuscript.

\section{CONFLICT OF INTEREST}

The authors have no conflict of interest to report.

\section{REFERENCES}

[1] Alzheimer Society of Canada (2016) Prevalence and Monetary Costs of Dementia in Canada. Alzheimer Society of Canada, Toronto.

[2] Galvin JE (2017) Prevention of Alzheimer's disease: Lessons learned and applied. J Am Geriatr Soc 65, 21282133.

[3] Cummings JL, Morstorf T, Zhong K (2014) Alzheimer's disease drug-development pipeline: Few candidates, frequent failures. Alzheimers Res Ther 6, 37.

[4] Mehta D, Jackson R, Paul G, Shi J, Sabbagh M (2017) Why do trials for Alzheimer's disease drugs keep failing? A discontinued drug perspective for 2010-2015. Expert Opin Investig Drugs 26, 735-739.

[5] Hsu D, A Marshall G (2017) Primary and secondary prevention trials in Alzheimer disease: Looking back, moving forward. Curr Alzheimer Res 14, 426-440.

[6] Cirrito JR, Yamada KA, Finn MB, Sloviter RS, Bales KR, May PC, Schoepp DD, Paul SM, Mennerick S, Holtzman DM (2005) Synaptic activity regulated interstitial fluid amyloid- $\beta$ levels in vivo. Neuron 48, 913-922.

[7] Iliff JJ, Wang M, Liao Y, Plogg BA, Peng W, Gundersen GA, Benveniste H, Vates GE, Deane R, Goldman SA (2012) A paravascular pathway facilitates CSF flow through the brain parenchyma and the clearance of interstitial solutes, including amyloid $\beta$. Sci Transl Med 4, 147ra111.

[8] Xie L, Kang H, Xu Q, Chen MJ, Liao Y, Thiyagarajan M, O'Donnell J, Christensen DJ, Nicholson C, Iliff JJ (2013) Sleep drives metabolite clearance from the adult brain. Science 342, 373-377.

[9] Van Someren EJ, Cirelli C, Dijk D-J, Van Cauter E, Schwartz S, Chee MW (2015) Disrupted sleep: From molecules to cognition. J Neurosci 35, 13889-13895.

[10] Mander BA, Winer JR, Jagust WJ, Walker MP (2016) Sleep: A novel mechanistic pathway, biomarker, and treatment target in the pathology of Alzheimer's disease? Trends Neurosci 39, 552-566.

[11] Yu J-T, Tan L, Hardy J (2014) Apolipoprotein E in Alzheimer's disease: An update. Annu Rev Neurosci 37, 79-100.

[12] Kanekiyo $\mathrm{T}, \mathrm{Xu} \mathrm{H}, \mathrm{Bu} G$ (2014) $A p o E$ and $\mathrm{A} \beta$ in Alzheimer's disease: Accidental encounters or partners? Neuron 81, 740-754.
[13] Shih I-F, Paul K, Haan M, Yu Y, Ritz B (2018) Physical activity modifies the influence of apolipoprotein $\mathrm{E} \varepsilon 4$ allele and type 2 diabetes on dementia and cognitive impairment among older Mexican Americans. Alzheimers Dement 14, $1-9$.

[14] Drogos LL, Gill SJ, Tyndall AV, Raneri JK, Parboosingh JS, Naef A, Guild KD, Eskes G, Hanly PJ, Poulin MJ (2016) Evidence of association between sleep quality and APOE $\varepsilon 4$ in healthy older adults A pilot study. Neurology 87, 18361842.

[15] Kahya M, Vidoni E, Burns JM, Thompson AN, Meyer K, Siengsukon CF (2017) The relationship between apolipoprotein $\varepsilon 4$ carrier status and sleep characteristics in cognitively normal older adults. J Geriatr Psychiatry Neurol 30, 273-279.

[16] Yesavage JA, Friedman L, Kraemer H, Tinklenberg JR, Salehi A, Noda A, Taylor JL, O'Hara R, Murphy G (2004) Sleep/wake disruption in Alzheimer's disease: APOE status and longitudinal course. J Geriatr Psychiatry Neurol 17, 20-24.

[17] Hita-Yañez E, Atienza M, Cantero JL (2013) Polysomnographic and subjective sleep markers of mild cognitive impairment. Sleep 36, 1327-1334.

[18] Lim AS, Yu L, Kowgier M, Schneider JA, Buchman AS, Bennett DA (2013) Sleep modifies the relation of APOE to the risk of Alzheimer disease and neurofibrillary tangle pathology. JAMA Neurol 70, 1544-1551.

[19] Spira AP, An Y, Peng Y, Wu MN, Simonsick EM, Ferrucci L, Resnick SM (2017) APOE genotype and nonrespiratory sleep parameters in cognitively intact older adults. Sleep $\mathbf{4 0}$, zsx076.

[20] Tsapanou A, Scarmeas N, Gu Y, Manly J, Schupf N, Stern Y, Barral S (2015) Examining the association between Apolipoprotein E (APOE) and self-reported sleep disturbances in non-demented older adults. Neurosci Lett 606, 72-76.

[21] Hillman CH, Erickson KI, Kramer AF (2008) Be smart, exercise your heart: Exercise effects on brain and cognition. Nat Rev Neurosci 9, 58-65.

[22] Bherer L, Erickson KI, Liu-Ambrose T (2013) A review of the effects of physical activity and exercise on cognitive and brain functions in older adults. J Aging Res 2013, 657508

[23] Erickson KI, Voss MW, Prakash RS, Basak C, Szabo A, Chaddock L, Kim JS, Heo S, Alves H, White SM, Wojcicki TR, Mailey E, Vieira VJ, Martin SA, Pence BD, Woods JA, McAuley E, Kramer AF (2011) Exercise training increases size of hippocampus and improves memory. Proc Natl Acad Sci U S A 108, 3017-3022.

[24] Brown AD, McMorris CA, Longman RS, Leigh R, Hill MD, Friedenreich CM, Poulin MJ (2010) Effects of cardiorespiratory fitness and cerebral blood flow on cognitive outcomes in older women. Neurobiol Aging 31, 2047-2057.

[25] Eskes GA, Longman S, Brown AD, McMorris CA, Langdon KD, Hogan DB, Poulin M (2010) Contribution of physical fitness, cerebrovascular reserve and cognitive stimulation to cognitive function in post-menopausal women. Front Aging Neurosci 2, 137.

[26] Davenport MH, Hogan DB, Eskes GA, Longman RS, Poulin MJ (2012) Cerebrovascular reserve: The link between fitness and cognitive function? Exerc Sport Sci Rev 40, 153-158.

[27] Jackson PA, Pialoux V, Corbett D, Drogos L, Erickson KI, Eskes GA, Poulin MJ (2016) Promoting brain health 
through exercise and diet in older adults: A physiological perspective. J Physiol 594, 4485-4498.

[28] Kline CE, Irish LA, Krafty RT, Sternfeld B, Kravitz HM, Buysse DJ, Bromberger JT, Dugan SA, Hall MH (2013) Consistently high sports/exercise activity is associated with better sleep quality, continuity and depth in midlife women: The SWAN sleep study. Sleep 36, 1279-1288.

[29] Montgomery P, Dennis JA (2002) Physical exercise for sleep problems in adults aged 60+. Cochrane Database Syst Rev, CD003404.

[30] Reid KJ, Baron KG, Lu B, Naylor E, Wolfe L, Zee PC (2010) Aerobic exercise improves self-reported sleep and quality of life in older adults with insomnia. Sleep Med 11, 934-940.

[31] Melancon MO, Lorrain D, Dionne IJ (2014) Exercise and sleep in aging: Emphasis on serotonin. Pathol Biol (Paris) 62, 276-283.

[32] Wilckens KA, Erickson KI, Wheeler ME (2016) Physical activity and cognition: A mediating role of efficient sleep. Behav Sleep Med 16, 569-586.

[33] Falck RS, Best JR, Davis JC, Liu-Ambrose T (2018) The independent associations of physical activity and sleep with cognitive function in older adults. J Alzheimers Dis 63, 1469-1484.

[34] Tyndall AV, Davenport MH, Wilson BJ, Burek GM, Arsenault-Lapierre G, Haley E, Eskes GA, Friedenreich CM, Hill MD, Hogan DB (2013) The brain-in-motion study: Effect of a 6-month aerobic exercise intervention on cerebrovascular regulation and cognitive function in older adults. BMC Geriatr 13, 21.

[35] Nasreddine ZS, Phillips NA, Bédirian V, Charbonneau $\mathrm{S}$, Whitehead V, Collin I, Cummings JL, Chertkow $\mathrm{H}$ (2005) The Montreal Cognitive Assessment, MoCA: A brief screening tool for mild cognitive impairment. J Am Geriatr Soc 53, 695-699.

[36] Corder E, Saunders AM, Risch N, Strittmatter W, Schmechel D, Gaskell P, Rimmler J, Locke P, Conneally P, Schmader K (1994) Protective effect of apolipoprotein E type 2 allele for late onset Alzheimer disease. Nat Genet 7, 180-184.

[37] Liu C-C, Kanekiyo T, Xu H, Bu G (2013) Apolipoprotein E and Alzheimer disease: Risk, mechanisms and therapy. Nat Rev Neurol 9, 106-118.

[38] Buysse DJ, Reynolds III CF, Monk TH, Berman SR, Kupfer DJ (1989) The Pittsburgh Sleep Quality Index: A new instrument for psychiatric practice and research. Psychiatry Res 28, 193-213.
[39] Johns MW (1991) A new method for measuring daytime sleepiness: The Epworth sleepiness scale. Sleep 14, 540545.

[40] Berry RB, Budhiraja R, Gottlieb DJ, Gozal D, Iber C, Kapur VK, Marcus CL, Mehra R, Parthasarathy S, Quan SF (2012) Rules for scoring respiratory events in sleep: Update of the 2007 AASM manual for the scoring of sleep and associated events: Deliberations of the sleep apnea definitions task force of the American Academy of Sleep Medicine. J Clin Sleep Med 8, 597-619.

[41] Pescatello LS, Arena R, Riebe D, Thompson PD (2014) American College of Sports Medicine's guidelines for exercise testing and prescription, 9th Ed. Wolters Kluwer/Lippincott Williams \& Wilkins, Philadelphia, PA.

[42] Abdi H (2010) Holm's sequential Bonferroni procedure. In Encyclopedia of Research Design, Salkind N, ed. Sage, Thousand Oaks, CA.

[43] Solomon A, Turunen H, Ngandu T, Peltonen M, Levälahti E, Helisalmi S, Antikainen R, Bäckman L, Hänninen T, Jula A, Laatikainen T, Lehtisalo J, Lindström J, Paajanen T, Pajala S, Stigsdotter-Neely A, Strandberg T, Tuomilehto J, Soininen H, Kivipelto M (2018) Effect of the apolipoprotein $\mathrm{E}$ genotype on cognitive change during a multidomain lifestyle intervention: A subgroup analysis of a randomized clinical trial. JAMA Neurol 75, 462-470.

[44] Buysse DJ, Hall ML, Strollo PJ, Kamarck TW, Owens J, Lee L, Reis SE, Matthews KA (2008) Relationships between the Pittsburgh Sleep Quality Index (PSQI), Epworth Sleepiness Scale (ESS), and clinical/polysomnographic measures in a community sample. J Clin Sleep Med 4, 563-571.

[45] Guadagni V, Burles F, Valera S, Hardwicke Brown E, Campbell T, Ferrara M, Iaria G (2016) The relationship between quality of sleep and emotional empathy. J Psychophysiol 31, 158-166.

[46] Landry GJ, Best JR, Liu-Ambrose T (2015) Measuring sleep quality in older adults: A comparison using subjective and objective methods. Front Aging Neurosci 7, 166.

[47] Surman C, Roth T (2011) Impact of stimulant pharmacotherapy on sleep quality: Post hoc analyses of 2 large, double-blind, randomized, placebo-controlled trials. J Clin Psychiatry 72, 903-908. 